\title{
Full-Field Three-Dimensional Volume Vibrations for Piezoceramic Materials in Resonance
}

\author{
Chien-Ching $\mathrm{Ma}^{*}$ \\ National Taiwan University, Taipei 106, Taiwan, Republic of China \\ Chi-Hung Huang ${ }^{\dagger}$ \\ Ching Yun Institute of Technology, Chung-Li 320, Taiwan, Republic of China \\ Hsien-Yang Lin ${ }^{\dagger}$ \\ De Lin Institute of Technology, Tu-Cheng 236, Taiwan, Republic of China \\ and \\ Chan-Chiao Lin \\ National Taiwan University, Taipei 106, Taiwan, Republic of China
}

\begin{abstract}
Three-dimensional volume vibrations for piezoceramics, which couple the out-of-plane and in-plane vibrations, are investigated by using an optical interferometry method called the amplitude-fluctuation electronic speckle pattern interferometry (AF-ESPI). The optical systems for AF-ESPI with out-of-plane and in-plane measurements are employed to analyze the volume vibration of piezoceramic material for a rectangular parallelepiped configuration. Because the clear fringe patterns measured by the AF-ESPI method will be shown only at resonant frequencies, both the resonant frequencies and the corresponding vibration mode shapes are successfully obtained at the same time. Two out-of-plane and two in-plane measurements are needed to construct the complete information of vibration mode shape. However, by tilting the specimen with an angle, the coupled out-of-plane and in-plane motions can be obtained from only one out-of-plane setup and measurement. The dominant motion of the volume vibration can be identified from the mode shape by tilting the specimen. Finally, the impedance analysis and finite element method with three-dimensional model are also utilized, and the results are compared with the measurements by AF-ESPI. Excellent agreements of the resonant frequencies and mode shapes are obtained from both results.
\end{abstract}

\section{Nomenclature}

$\begin{array}{ll}A(t) & =\text { amplitude of out-of-plane vibration } \\ A^{\prime}(t) & =\text { amplitude of in-plane vibration } \\ I_{O} & =\text { object light intensity } \\ I_{R} & =\text { reference light intensity } \\ J_{n} & =\text { Bessel function of the first kind of order } n \\ J_{0} & =\text { zeroth-order Bessel function of the first kind } \\ t & =\text { time } \\ x_{1}, x_{2}, x_{3} & =\text { Cartesian coordinate system } \\ \theta & =\text { angle between object light and observation } \\ \theta^{\prime} & =\text { direction } \\ \lambda & =\text { wavelength of laser } \\ \tau & =\text { charge-coupled device refreshing time } \\ \phi & =\text { phase difference between reference and object light } \\ \omega & =\text { angular frequency }\end{array}$

\section{Introduction}

W HEN a coherent laser beam illuminates a rough surface, reflected coherent wavelets from each point of the rough surface will form a random pattern of laser speckles and fill the entire space. This is known as the speckle effect. The random speckle distribution is a function of spatial coordinates and is an information carrier, which can be used in many engineering applications(surface

Received 19 April 2002; accepted for publication 19 September 2002. Copyright (C) 2002 by the American Institute of Aeronautics and Astronautics, Inc. All rights reserved. Copies of this paper may be made for personal or internal use, on condition that the copier pay the $\$ 10.00$ per-copy fee to the Copyright Clearance Center, Inc., 222 Rosewood Drive, Danvers, MA 01923 ; include the code 0001-1452/03 $\$ 10.00$ in correspondence with the CCC.

*Professor, Department of Mechanical Engineering; cma@w3.me.ntu. edu.tw.

${ }^{\dagger}$ Associate Professor, Department of Mechanical Engineering.

* Graduate Student, Department of Mechanical Engineering. roughness measurement, deformation and displacement measurement, and vibration analysis, etc.). ${ }^{1}$ Using a standard television camera to digitize the speckles and to record the fringe patterns would eliminate the use of photographic film. This method is known as the electronic speckle pattern interferometry(ESPI). ESPI is a technique that uses a laser, a charge-coupled device (CCD) camera, and a digital processor to generate interferograms. The most common light source for ESPI is a continuous wave laser because of its lower price and optical power requirement. As a result of ESPI's use of video recording, its real-time nature makes it practical for vibration measurement in experiments.

The basic principles of ESPI were first proposed by Butters and Leendertz ${ }^{2}$ to investigate the transverse vibration of a disk. This technique (also called TV holography or electronic holography) is a full-field, noncontact, and real-time method to measure the deformation for structures subjected to various kinds of loading. Since then many researchers have been steered toward the area of the deformation analysis, especially for vibration measurements. As compared with traditional holographicinterferometry, ${ }^{3}$ the cumbersome and time-consuming chemical development can be eliminated for ESPI, and the experimental process will be sped up. Because only $1 / 30 \mathrm{~s}$ is needed to record and update a frame of interferometric pattern, ESPI is faster in operation and more insensitive to enviroment than holography. The most widely used experimental setup to study dynamic responses by ESPI is the time-averaged vibration ESPI method. ${ }^{4}$ When the laser light source is employed, time-averaged interferometric fringes are produced for the harmonically vibrating object, which offers a good observation of the vibration mode shape. The disadvantage of this method is that the interferometry fringes represent only the amplitude but not the phase of the vibration. To improve this shortcoming, the phase-modulation method using reference beam modulation technique was developed to determine the relative phase of the displacement. ${ }^{5}$ In addition to the method already mentioned, a number of variations for the principles based on the ESPI technique were proposed in many academic researches and engineering applications. Creath and Slettemoen ${ }^{6}$ 
developed the subtraction method to reduce the noise coming from the environment. The subtraction method differs from the timeaveraged method in its reference frame that is first recorded before vibration and continuously subtracted from the incoming frames after vibration. In $1985, \mathrm{~L} \mathrm{kberg}^{7}$ indicated that in-plane vibration modes could be obtained by using an out-of-plane setup and tilting the specimen with an angle. Tilting angles between 60 and $70 \mathrm{deg}$ would be an acceptablecompromise between distortion and sensitivity. Shellabear and Tyrer ${ }^{8}$ extended the time-averagedESPI to make three-dimensional vibration measurements. Doval et al. ${ }^{9}$ proposed the additive stroboscopic TV holography for out-of-plane vibration analysis, which exhibited an enhanced contrast with constant visibility fringes and dynamic phase shifting. To increase the visibility of the fringe pattern, an amplitude-fluctuation method was proposed by Wang et al. ${ }^{10}$ for out-of-plane vibration measurement. The reference frame in the amplitude-fluctuation method is taken in a vibrating state, and both the visibility and resolution of the fringe pattern are better than those obtained by other time-averaged methods.

Piezoelectric materials are widely used in electromechanicalsensors, actuators, ${ }^{11}$ and nondestructive testing, as well as electrooptic modulator, etc. Researches and development activities in these materials are now intense and widespread. The piezoelectric effect was discovered in 1880 by Pierre and Jacques Curie and has been addressed in later literature (Berlincourt et al. ${ }^{12}$ and Zelenka ${ }^{13}$ ). Piezoelectric effect is applied to many modern engineering applications because of the coupling between the electrical and mechanical fields. Although the vibration characteristics of piezoelectric materials can be determined by three-dimensional equations of the linear elasticity, the Maxwell equation, and piezoelectric constitutive equations, ${ }^{14}$ it is difficult to obtain analytical solutions even for a simple geometry. On account of the complication just mentioned, the variational approximation method and finite element analysis are usually employed to study the vibrating problem of piezoelectric materials. Kharouf and Heyliger ${ }^{15}$ used the RayleighRitz method to solve the static and axisymmetric vibration problems for piezoelectric disks, hollow cylinders, and composite cylinders. Heyliger ${ }^{16}$ used the discrete-layer model, accounting for the discontinuity in slope across interfaces, to investigate free vibration of layered elastic-piezoelectric parallelepipeds. The variation method is a powerful and accurate technique for simple geometries, and the finite element method is an alternative method to the analysis of piezoelectric material in various complicated configurations. Adelman and Stavsky ${ }^{17}$ derived the electroelasticequationsfor solving the axisymmetric vibrations of radially polarized piezoelectric homogeneous ceramics tubes. Influences coming from boundary conditions and the ratio of inner/outer radii are discussed in details. Then they extended the method to study the problems of radially polarized composite piezoelectric cylinders and disks. ${ }^{18}$ Heyliger and Ramire ${ }^{19}$ introduced a numerical model for the study of computing natural frequencies of layered elastic and piezoelectriccylinders layered in the thicknessdirection. The method combines approximations of one-dimensional finite elements in the thickness direction and analytic functions in the plane. Migliori and Sarrao ${ }^{20}$ provided many successful applications of the vibrational resonances in engineering systems.

In addition to variational and numerical methods, experimental technique has been employed for investigation of vibration modes and natural frequencies of piezoelectric material. Shaw ${ }^{21}$ used an optical interference technique in which a stroboscopically illuminated multiple beam was applied to measure the surface motion of thick barium titanate disks. However, only normal modes having symmetry with respect to the axis and to the central plane were observed. Koyuncu ${ }^{22}$ used ESPI with reference beam modulation to observe the vibration amplitudes and vibration modes of PZT-4 transducers in air and water. Ma and Huang ${ }^{23}$ and Huang and $\mathrm{Ma}^{24}$ used the amplitude-fluctuation-ESPIAF-ESPI method to investigate the three-dimensional vibration of piezoelectric rectangular parallelepipedscylinders, respectively; both the resonant frequenciesand mode shapes were presented.

To obtain the reliable dynamic response of piezoceramic materials, it is necessary to visibly examine the vibration response from an experimental point of view so that the characteristics of the materi- als can be elucidated thoroughly. The experimental measurement of the resonant frequencies for the piezoceramic material is generally performed by impedance analysis. However, a prominent feature of utilizing piezoceramic materials lies in the capability of being investigated for their full-field and real-time vibration behavior. In this paper a full-field experimental method based on the AF-ESPI is used to study the three-dimensional vibration of a rectangular parallelepiped piezoceramic. Both in-plane and out-of-plane motions in each face of the rectangular parallelepipedare obtained and discussed. By tilting the specimen with a proper angle, the vibration modes that couple out-of-plane and in-plane motions are also investigated. It is easy to see the complete three-dimensional volume vibration modes and the dominant motion from the tilted specimen. The numerical finite element method with three-dimensional model and experimental impedance analysis are also used to study the problem, and the results are compared with those obtained by AF-ESPI. Excellent agreements of the resonant frequencies and mode shapes are obtained for experimental measurements and numerical results.

\section{AF-ESPI for Out-of-Plane and In-Plane Vibration Measurements}

The most familiar way of ESPI used for vibration analysis is the time-averaged method with an image sensor (most commonly used is a CCD array) integrating the speckle interferogram field pixel by pixel. The name "time-averaged" denotes that the vibration measurement includes many periods of object motions during the camera frame period. Two different optical setups are used for the vibration measurement in this study.

\section{Out-of-Plane Vibration}

The optical setup of ESPI for out-of-plane measurement is shown in Fig. 1. At a given instant time $t$, the light intensity in the image plane detected by a CCD camera can be expressed $\mathrm{as}^{25}$

$$
I(t)=I_{R}+I_{O}+2 \sqrt{I_{R} I_{O}} \cos [\phi+(2 \pi / \lambda)(1+\cos \theta) A(t)]
$$

In the time-averaged method the light intensity is averaged over the CCD refreshing time $\tau$ to obtain

$$
I_{\tau}=I_{R}+I_{O}+\frac{2 \sqrt{I_{R} I_{O}}}{\tau} \int_{0}^{\tau} \cos \left[\phi+\frac{2 \pi}{\lambda}(1+\cos \theta) A(t)\right] \mathrm{d} t
$$

In the vibration measurement the intensity average $I_{\tau}$ is evaluated for sinusoidal vibration, that is, $A(t)=A \cos \omega t$. Let

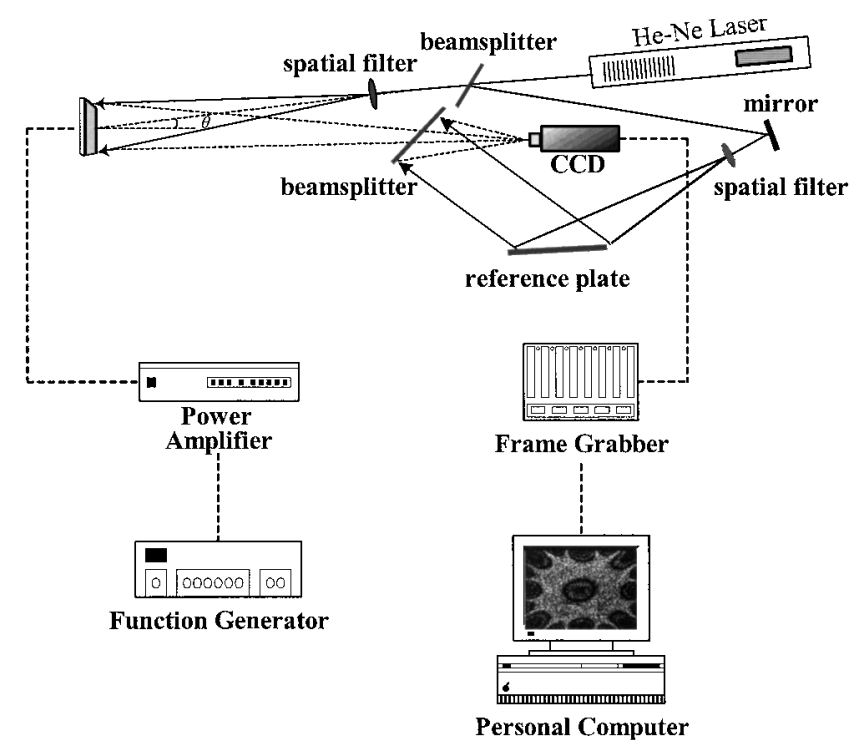

Fig. 1 Schematic diagram of AF-ESPI setup for the out-of-plane measurement. 
$\Gamma=(2 \pi / \lambda)(1+\cos \theta)$ and $\tau=2 m \pi / \omega$, where $m$ is an integer. Then Eq. (2) can be written as

$$
\begin{aligned}
I_{\tau}= & I_{R}+I_{O}+\frac{2 \sqrt{I_{R} I_{O}}}{\tau} \int_{0}^{\tau} \cos (\phi+\Gamma A \cos \omega t) \mathrm{d} t \\
& =I_{R}+I_{O}+\frac{2 \sqrt{I_{R} I_{O}}}{\tau} \operatorname{Re}\left\{e^{i \phi} \int_{0}^{\tau} \exp (i \Gamma A \cos \omega t) \mathrm{d} t\right\}
\end{aligned}
$$

where $R e$ stands for the real part. Because

$$
e^{i z \sin \alpha}=\sum_{n=-\infty}^{\infty} J_{n}(z) e^{i n \alpha}
$$

we have

$$
I_{\tau}=I_{R}+I_{O}+\frac{2 \sqrt{I_{R} I_{O}}}{\tau} \operatorname{Re}\left\{e^{i \phi} \sum_{n=-\infty}^{\infty} J_{n}(\Gamma A) \int_{0}^{\tau} e^{i n(\pi / 2-\omega t)} \mathrm{d} t\right\}
$$

However, all of the terms will be zero except $n=0$; finally, Eq. (2) can be expressed as

$$
I_{\tau}=I_{R}+I_{O}+2 \sqrt{I_{R} I_{O}}(\cos \phi) J_{0}(\Gamma A)
$$

After image processing and rectifying, the intensity of the first image, named the reference image, can be expressed as

$$
I_{\tau}=I_{R}+I_{O}+2 \sqrt{I_{R} I_{O}}\left|(\cos \phi) J_{0}(\Gamma A)\right|
$$

As the vibration of the specimen continues, we assume that the vibration amplitude changes from $A$ to $A+\Delta A$ because of the electronic noise or instability of the apparatus. The light intensity of the second image $I_{\tau}^{\prime}$ can be represented as

$$
I_{\tau}^{\prime}=I_{R}+I_{O}+\frac{2 \sqrt{I_{R} I_{O}}}{\tau} \int_{0}^{\tau} \cos [\phi+\Gamma(A+\Delta A) \cos \omega t] \mathrm{d} t
$$

When the vibration amplitude variation $\Delta A$ is rather small, Eq. (5) can be expanded in Taylor series. By keeping the first two terms and neglecting the higher-order terms, we rewrite Eq. (5) as follows:

$$
I_{\tau}^{\prime}=I_{R}+I_{O}+2 \sqrt{I_{R} I_{O}}(\cos \phi)\left[1-\frac{1}{4} \Gamma^{2}(\Delta A)^{2}\right] J_{0}(\Gamma A)
$$
as

By image processing and rectifying, $I_{\tau}^{\prime}$ can be similarly expressed

$$
I_{\tau}^{\prime}=I_{R}+I_{O}+2 \sqrt{I_{R} I_{O}}\left|(\cos \phi)\left[1-\frac{1}{4} \Gamma^{2}(\Delta A)^{2}\right] J_{0}(\Gamma A)\right|
$$

When these two images (the reference and second images) are subtracted by the image processing system, that is, Eq. (4) is subtracted from Eq. (7), and are rectified, the resulting image intensity can be expressed as

$$
I=I_{\tau}^{\prime}-I_{\tau}=\left(\sqrt{I_{R} I_{O}} / 2\right)\left|(\cos \phi) \Gamma^{2}(\Delta A)^{2} J_{0}(\Gamma A)\right|
$$

\section{In-Plane Vibration}

The optical setup of the AF-ESPI system for in-plane vibration measurementis shown in Fig. 2. Similar to the out-of-planevibration measurement, the reference and second image intensities, that is, $I_{\tau}$ and $I_{\tau}^{\prime}$, for in-plane vibration are expressed as

$$
\begin{gathered}
I_{\tau}=2 I_{O}+2 I_{O}\left|(\cos \phi) J_{0}\left(\Gamma^{\prime} A^{\prime}\right)\right| \\
I_{\tau}^{\prime}=2 I_{O}+2 I_{O}\left|(\cos \phi)\left[1-\frac{1}{4} \Gamma^{\prime 2}\left(\Delta A^{\prime}\right)^{2}\right] J_{0}\left(\Gamma^{\prime} A^{\prime}\right)\right|
\end{gathered}
$$

where $\Gamma^{\prime}=(2 \pi / \lambda)\left(2 \sin \theta^{\prime}\right)$. Subtracting Eq. (10) from Eq. (9) and rectifying by the image processing system, we can obtain the resulting image intensity as

$$
I=I_{\tau}^{\prime}-I_{\tau}=\left(I_{O} / 2\right)\left|(\cos \phi) \Gamma^{\prime 2}\left(\Delta A^{\prime}\right)^{2} J_{0}\left(\Gamma^{\prime} A^{\prime}\right)\right|
$$

Combining the out-of-plane with the in-plane optical setups by the AF-ESPI method, we can construct completely the threedimensional volume vibration characteristics of the piezoceramic
Table 1 Material properties of the PZT-4 piezoceramics

\begin{tabular}{lc}
\hline \hline Quantity & PZT-4 \\
\hline$c_{11}^{E}\left(10^{10} \mathrm{~N} / \mathrm{m}^{2}\right)$ & 13.9 \\
$c_{33}^{E}$ & 11.54 \\
$c_{12}^{E}$ & 7.784 \\
$c_{13}^{E}$ & 7.428 \\
$c_{44}^{E}$ & 2.564 \\
$c_{66}^{E}=\left(c_{11}^{E}-c_{12}^{E}\right) / 2$ & 3.058 \\
$e_{31}(\mathrm{~N} / \mathrm{Vm})$ & -5.2 \\
$e_{33}$ & 15.08 \\
$e_{15}$ & 12.72 \\
$\varepsilon_{11}^{S} / \varepsilon_{0}$ & 762 \\
$\varepsilon_{33}^{S} / \varepsilon_{0}$ & 663 \\
$\rho, \mathrm{kg} / \mathrm{m}^{3}$ & 7500 \\
$\varepsilon_{0}, \mathrm{~F} / \mathrm{m}$ & $8.85 \times 10^{-12}$ \\
\hline \hline
\end{tabular}

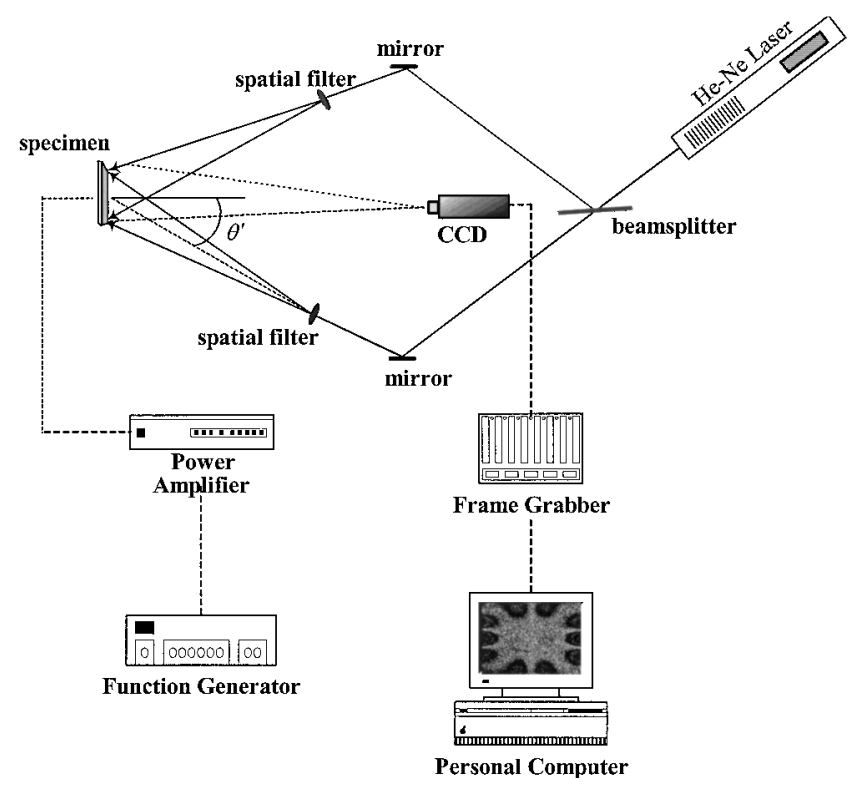

Fig. 2 Schematic diagram of AF-ESPI setup for the in-plane measurement.

material, including resonant frequencies and mode shapes at the same time. This is different from the conventional impedance analysis, which has been used widely in determining only the resonant frequency for piezoceramic material. There are also very few experimental techniques with the ability to obtain the three-dimensional mode shapes.

\section{Experimental and Numerical Results}

A rectangularparallelepiped piezoceramic, made of PZT-4, is selected for experimental and numerical investigations of the volume vibration characteristics in resonance. The material properties and the geometric configurations of the specimen are shown in Table 1 (Ref. 26) and Fig. 3, respectively. The polarization axis is in the $x_{3}$ direction, and two opposite faces ( $x_{1}-x_{2}$ plane) of the specimen are completely coated with silver electrodes. The piezoceramic block is excited by the application of an ac voltage across electrodes on the surfaces and has completely stress-free boundary conditions.

The schematic layout of self-arranged time-averaged AF-ESPI optical systems, as shown in Figs. 1 and 2, are employed to perform the out-of-plane and in-plane experimental measurements for resonant frequencies and corresponding mode shapes. An He-Ne laser with $30 \mathrm{~mW}$ and wavelength $\lambda=632.8 \mathrm{~nm}$ is used as the coherent light source. As shown in Fig. 1 for the out-of-plane measurement, the laser beam is split into two parts, the object and reference beam, by a beamsplitter. We use a CCD camera (Pulnix Co.) and a P360F (Dipix Technologies, Inc.) frame grabber with a digital signal processor onboard to record and process the images. The object beam travels to the piezoceramic specimen and then reflects to the $\mathrm{CCD}$ 

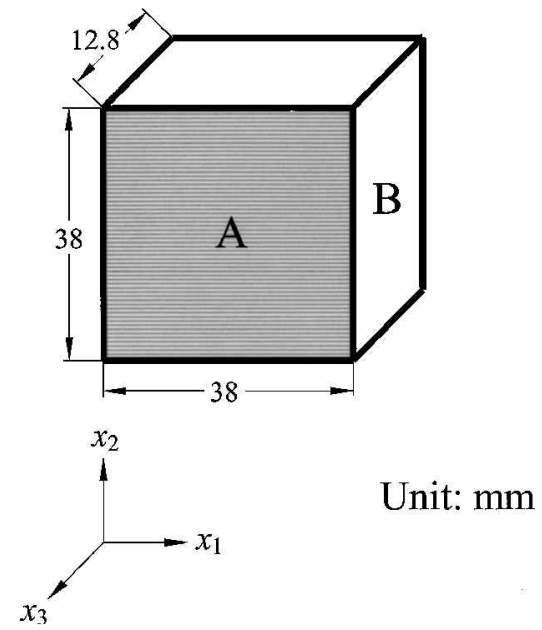

Fig. 3 Geometric dimensions of the rectangular parallelepiped piezoceramics.

camera. The reference beam goes directly to the CCD camera via a mirror and a reference plate. The object and reference beams are combined into the CCD sensor array through a zoom lens. The optical path length and the light intensities of these two beams should remain identical in the experimental setup for better contrast of the interferometric fringes. For the in-plane measurement system as shown in Fig. 2, two laser beams with the same optical path and light intensity are symmetrically incident to the specimen, and then reflect to the $C C D$ camera. Once the specimen is vibrating, the interferogram recorded by the CCD camera is stored in an image buffer as a reference image, and then the next frame is grabbed and subtracted by the image processing system. The CCD camera converts the intensity distribution of the interference pattern of the object into a corresponding video signal at $30 \mathrm{frames} / \mathrm{s}$, which is in consequence processed electronically and finally converted into an image on the video monitor. The interpretation of the fringe image is similar to the reading of a displacement contour. To achieve a sinusoidal output, a digitally controlled function generator (Hewlett-Packard HP33120A) connected to a power amplifier (NF Electronic Instruments Type 4005) is employed as an input source, which generates a periodical exciting force to the specimen. The size of the specimen that can be used in the experimental measurement is dependent on the optical system, the power of laser and the zoom lens. For the experimental setup as mentioned, the suitable dimension of the specimen is ranged from 1 to $100 \mathrm{~cm}^{2}$ and is able to obtain good quality of fringe patterns.

Detailed experimental procedures of the AF-ESPI technique for both the in-plane and the out-of-plane vibrations are performed as follows. First, a reference image is taken after the specimen vibrates with the second image taken subsequently; the reference image is subtracted by the image processing system. If the vibrating frequency is not the resonant frequency, only randomly distributed speckles are displayed, and no fringe patterns will be shown. In case the vibrating frequency falls in the neighborhood of the resonant frequency, distinct stationary fringe patterns will be observed in the monitor. Then, the function generator is carefully and gradually turned; the number of fringes will increase, and fringe pattern will become clearer as the resonant frequency is approached. From the aforementioned experimental procedure the resonant frequencies and the correspondent mode shapes can be determined at the same time by using the AF-ESPI optical system.

When the rectangularparallelepipedvibrates at resonant frequencies, out-of-plane and in-plane motions are coupled and are induced in each face. From the out-of-plane and in-plane AF-ESPI optical systems used in this study, the out-of-plane and in-plane motion can be decoupled. Figures 4-10 show the experimental and numerical results of the first seven volume vibration mode shapes of the specimen, with both the out-of-plane and in-plane motions displayed in each face. The displacement in the $x_{3}$ direction of the rectangular parallelepiped as shown in Fig. 3 will be denoted as the out-of-plane motion in face A and the in-plane motion in face B. Similarly, the
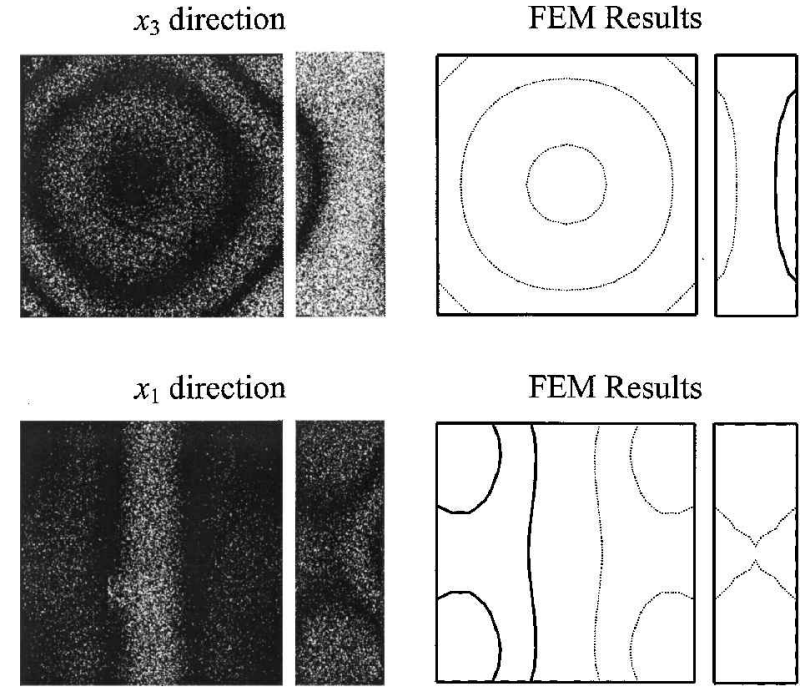

FEM Results

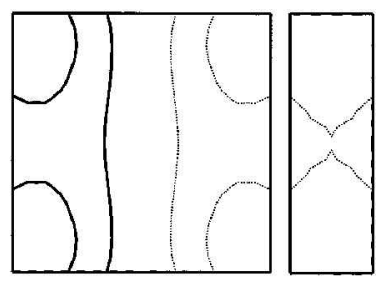

Tilt $60^{\circ}$

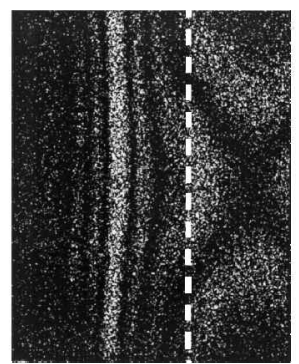

Fig. 4 First mode shape of the specimen obtained by AF-ESPI and FEM $(47.57 \mathrm{kHz})$. $x_{3}$ direction

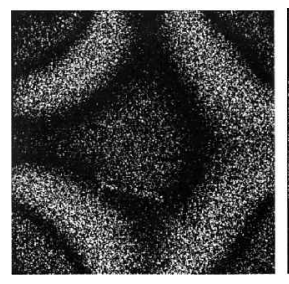

$x_{1}$ direction

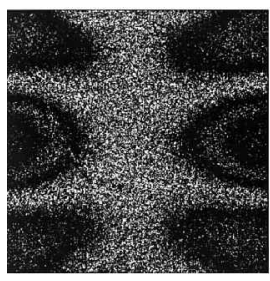

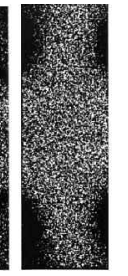

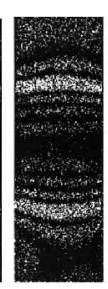

FEM Results

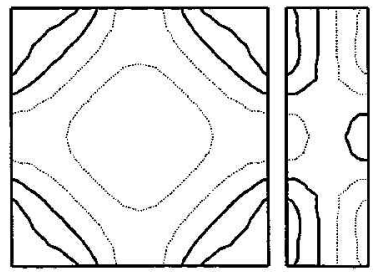

FEM Results

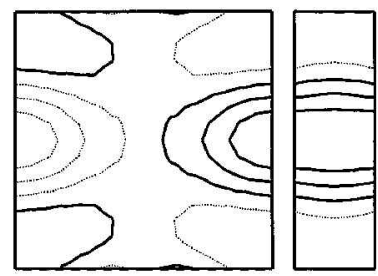

Tilt $60^{\circ}$

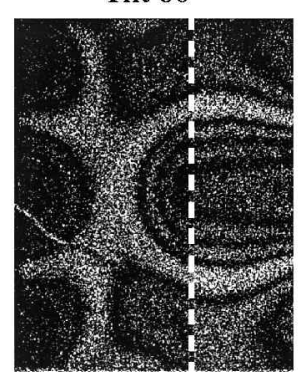

Fig. 5 Second mode shape of the specimen obtained by AF-ESPI and FEM (71.84 kHz). 
$x_{3}$ direction
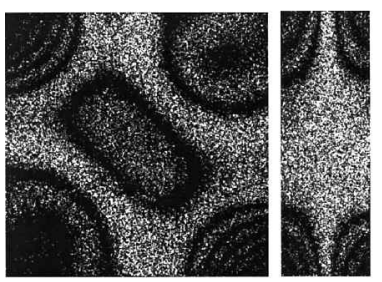

$x_{1}$ direction
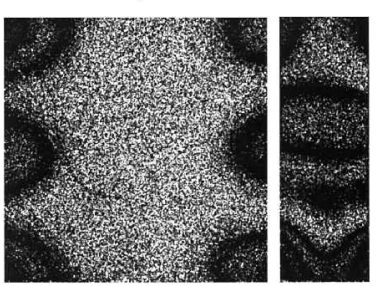

$x_{3}$ direction

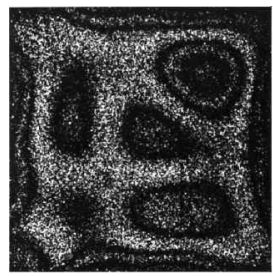

$x_{1}$ direction

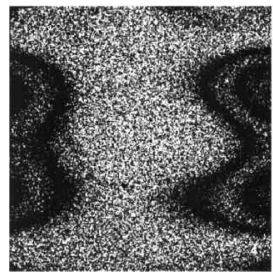

FEM Results
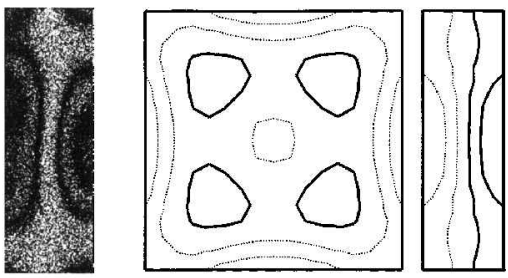

FEM Results
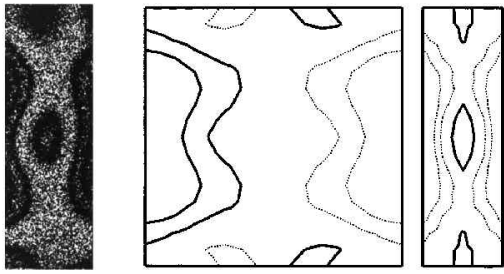

Tilt $60^{\circ}$

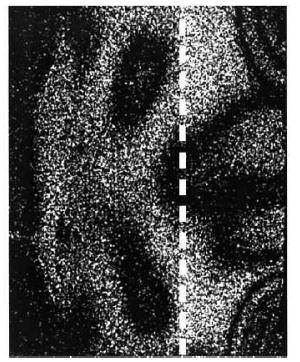

Fig. 6 Third mode shape of the specimen obtained by AF-ESPI and FEM (104.56 kHz).
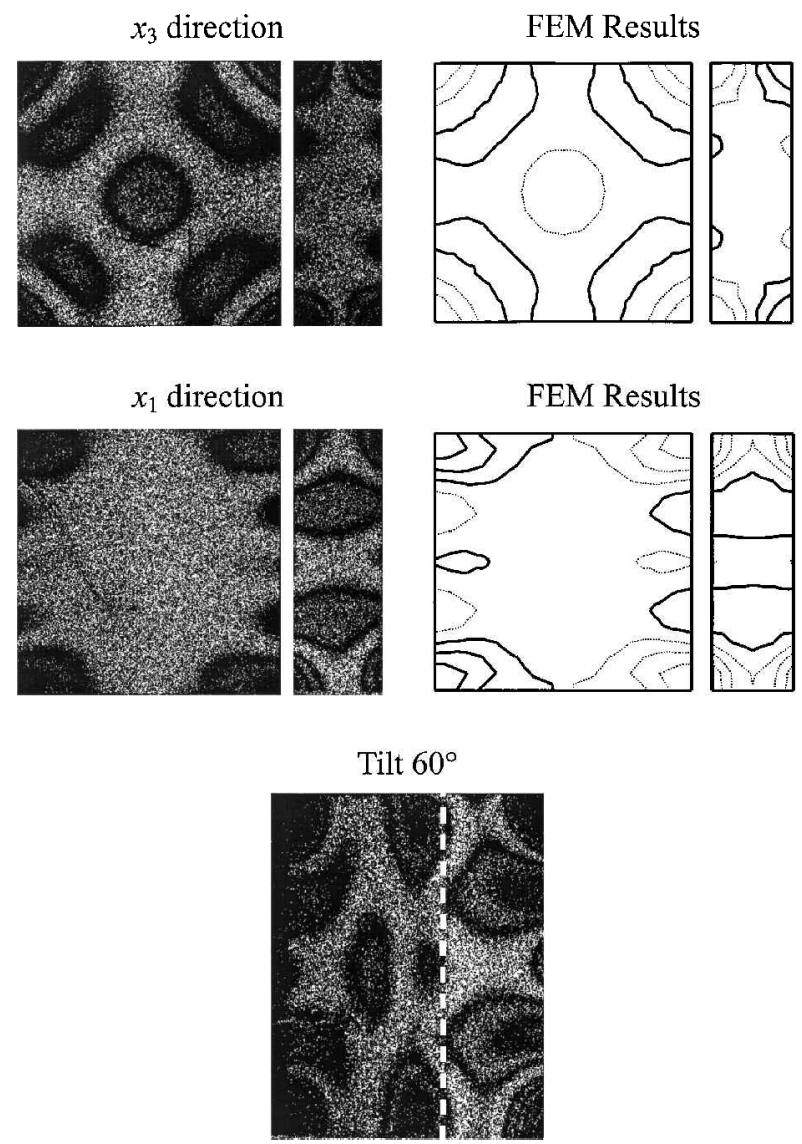

Fig. 7 Fourth mode shape of the specimen obtained by AF-ESPI and FEM $(109.63 \mathrm{kHz})$.

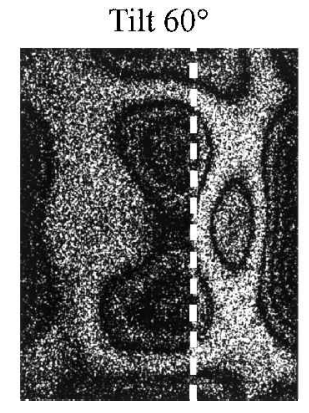

Fig. 8 Fifth mode shape of the specimen obtained by AF-ESPI and FEM (116.2 kHz).
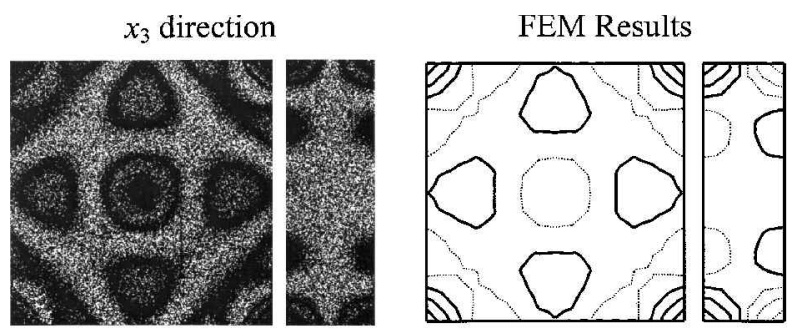

$x_{1}$ direction

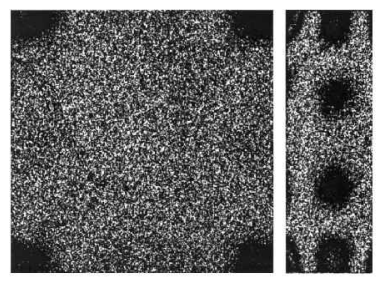

FEM Results

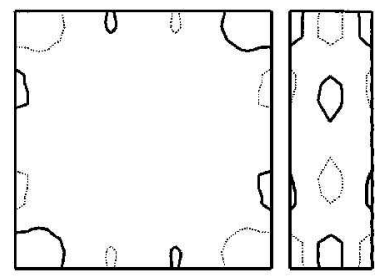

Tilt $60^{\circ}$

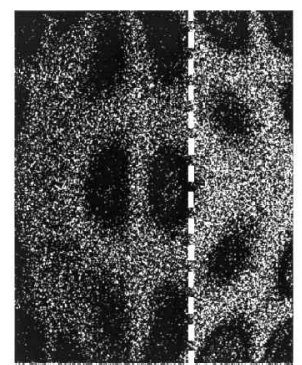

Fig. 9 Sixth mode shape of the specimen obtained by AF-ESPI and FEM (126.24 kHz) 
$x_{3}$ direction
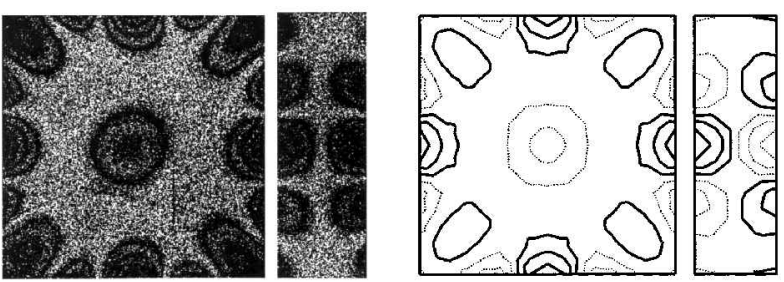

$x_{1}$ direction
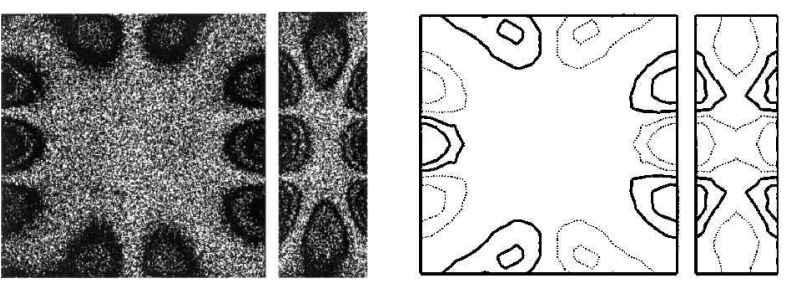

Tilt $60^{\circ}$

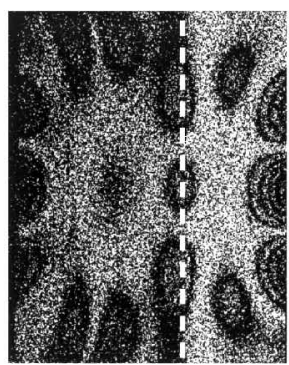

Fig. 10 Seventh mode shape of the specimen obtained by AF-ESPI and FEM (138.8 kHz).

displacement in the $x_{1}$ direction will be denoted as the in-plane motion and the out-of-plane motion in face A and face B, respectively. In addition, in light of the results presented in this work numerical calculation is also investigated by the commercially available software ABAQUS finite element package ${ }^{27}$ in which a 20-node three-dimensionalbrick element (C3D20E) and 1734 elements are selected to analyze the problem. As shown in finite element results presented in Figs. 4-10, mode shapes of face A for out-of-plane motion match with those of face $B$ for in-plane motion on the edge between faces A and B. We can see that experimental results are in good agreement with numerical calculations by finite element method. We indicate the phase of displacement in finite element results as solid or dashed lines; the solid lines are in the opposite direction to the dashed lines. The transition from solid lines to dashed lines corresponds to a zero displacement line or a nodal line. The zero-order fringe, which is the brightest fringes on experimental results, represents the nodal lines of the vibrating piezoceramics at resonant frequencies. It is noted that four measurements, that is, two out-of-plane and two in-plane motions, are needed to construct a complete vibration mode shape.

To obtain the three-dimensionalvibrationmode shape in one measurement, we rotate the specimen clockwise about the $x_{2}$ axis with an angle $\eta=60 \mathrm{deg}$ and measure the mode shapes by using the out-of-plane setup. The experimental fringe patterns of the tilted specimen are also presented in the bottom of Figs. 4-10; the white dashed line is plotted in the specimen to indicate the edge between face A and B. Figure 11 shows the specimen arrangement and the measurement direction (out-of-plane or in-plane) in this study; each configuration corresponds to the experimental results presented in Figs. 4-10. After the specimen is tilted with an angle, the displacement in the $x_{3}$ direction (or the observation direction) can be expressed as $u=u_{1} \sin \eta+u_{3} \cos \eta$, and the observed mode shapes are coupled with in-plane and out-of-plane motions. Although the mode shape in three-dimensional configuration is coupled with out-of-plane and in-plane motions, some modes are dominated by
Table 2 Relative maximum displacements for out-of-plane and in-plane motions

\begin{tabular}{lccr}
\hline \hline Mode & $\begin{array}{c}\text { AF-ESPI, } \\
\mathrm{kHz}\end{array}$ & $\begin{array}{c}\text { Impedance } \\
\text { analysis, kHz }\end{array}$ & $\begin{array}{c}\text { FEM, } \\
\mathrm{kHz}\end{array}$ \\
\hline 1 & 47.57 & 47.7 & 49.10 \\
2 & 71.84 & 71.7 & 71.37 \\
3 & 104.56 & 104.7 & 105.67 \\
4 & 109.63 & 109.8 & 110.21 \\
5 & 116.2 & 116.1 & 117.33 \\
6 & 126.24 & 126.3 & 126.71 \\
7 & 138.8 & 139.2 & 140.76 \\
\hline \hline
\end{tabular}
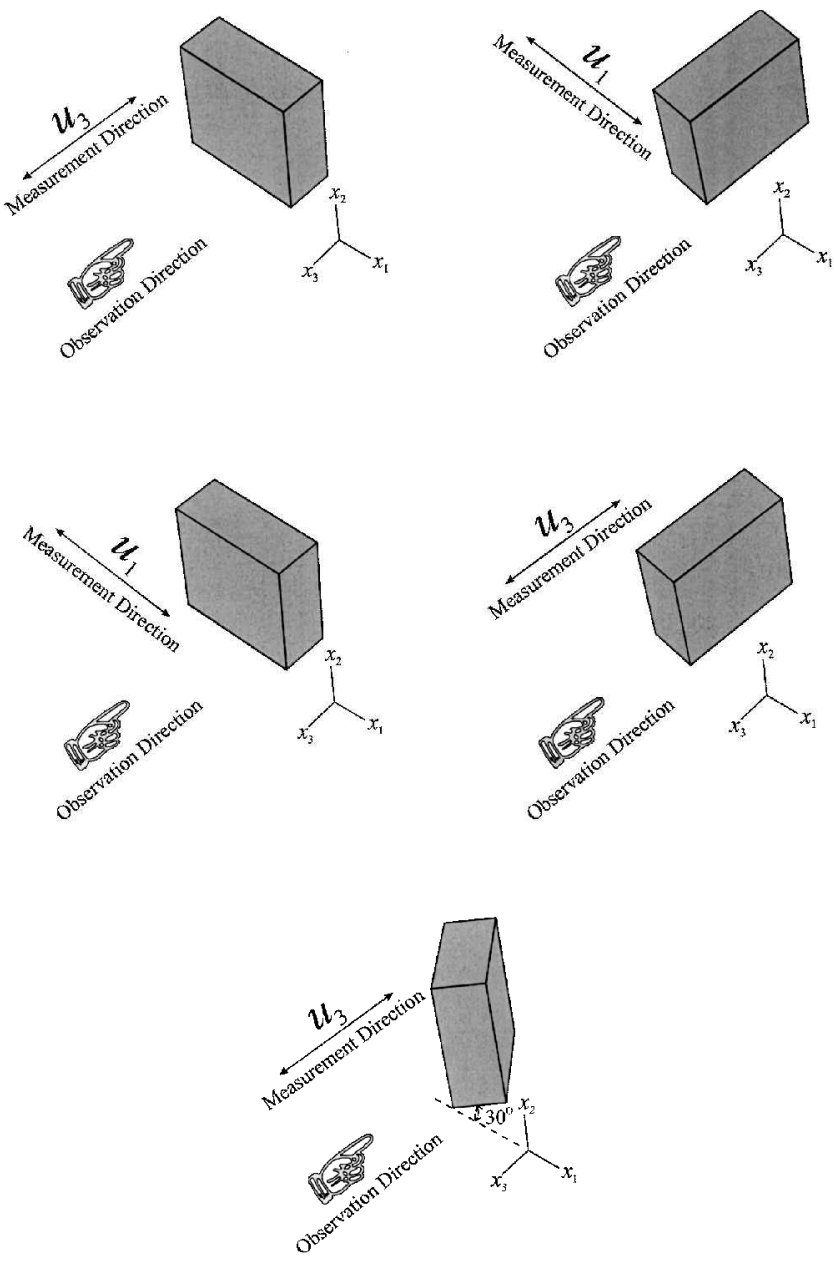

Fig. 11 Experimental configuration for the specimen arrangements and measurement directions.

out-of-plane or in-plane motions. Table 2 shows the relative displacements for out-of-plane and in-plane motions. We can see that modes 1, 2, and 7 are dominated by in-plane motions. It is also evident in Figs. 4-10 that the mode shapes of the tilted specimen for modes 1,2 , and 7 are similar to the pure in-plane motions. It can be concluded that if the in-plane motion is dominated, the in-plane mode shape can be obtained from the out-of-plane setup and by tilting the specimen.

Because the electrical impedance of the piezoceramic material drops to a local minimum when piezoceramics vibrates at a resonant frequency, the resonant frequency can also be determined by the impedance analysis. Herein, it is carried out by using HP4194A impedance/gain-phase analyzer (Hewlett Packard), and the experimental setup is shown in Fig. 12. Table 3 shows the resonant frequencies of rectangularparallelepipedpiezoceramics obtained from AF-ESPI, impedance analysis, and finite element method (FEM). The differences between the experimental data and FEM might result from the measurement of material properties and the faults of specimens that are generated by manufacturing process. 
Table 3 Results of resonant frequencies obtained from AF-ESPI, impedance analysis, and FEM for the rectangular parallelepiped piezoceramics

\begin{tabular}{llll}
\hline \hline \multirow{2}{*}{ Mode } & \multicolumn{3}{c}{ Max. normalized amplitude $\left(A / A_{\max }\right)$} \\
\cline { 2 - 4 } 1 & \multicolumn{1}{c}{$x_{1}$} & 1 & $x_{3}$ \\
\hline 2 & 1 & 1 & 0.6475 \\
3 & 1 & 0.4656 & 0.3213 \\
4 & 0.4656 & 0.5474 & 1 \\
5 & 0.5474 & 0.8615 & 1 \\
6 & 0.8615 & 0.3838 & 1 \\
7 & 0.3838 & 1 & 0.8639 \\
\hline \hline
\end{tabular}

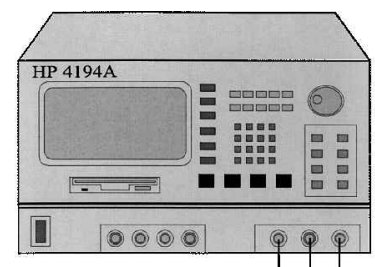

Impedance Analyzer

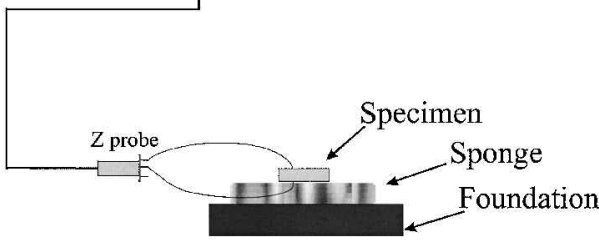

Fig. 12 Experimental setup for the impedance analyzer.

\section{Conclusions}

Optical techniques have been shown to have certain advantages for vibration analysis, and ESPI has been applied to many vibration problems. The advantages of the optical ESPI method include noncontact and full-field measurement, real-time observation, submicrometer sensitivity, and digital image processing, among others. As compared with the film recording and optical reconstruction procedures used for holographic interferometry, the interferometric fringes of AF-ESPI are produced instantly by a video recording system.

Vibration characteristics of piezoelectric materials are important in many engineering applications. Most of the works for vibration analysis of piezoelectric plates published in the literature are analytical and numerical results for two-dimensional problems. There are only few experimentalresults availablefor the three-dimensional full-field configuration of mode shapes for volume vibrations. In this study two self-arranged amplitude-fluctuation ESPI optical setups have been established to obtain the resonant frequencies and the corresponding mode shapes of vibrating piezoceramic material for a rectangular parallelepiped configuration. Both the in-plane and out-of-plane vibration mode shapes on each face of the specimen are obtained, and the results are well compared with numerical finite element calculations. The coupled out-of-plane and in-plane motions are obtained from the out-of-planesetup by tilting the specimen with an angle. The dominant motion of the volume vibration is identified and discussed in detail. The resonant frequencies of the piezoelectric materials are usually determined experimentally by the impedance analysis, which is also performed in this study. The resonant frequencies obtained by AF-ESPI method are in good agreement with the impedance analysis. Excellent agreements between the experimentaldata and numerical resultsreveal that the presented methodologies are capable of measuring vibration response of piezoceramic materials with satisfactory accuracy.

\section{Acknowledgment}

The authors gratefully acknowledge the financial support of this research by the National Science Council (Republic of China) under Grant NSC 90-2212-E-002-163.

\section{References}

${ }^{1}$ Erf, R. K., Speckle Metrology, Academic Press, New York, 1978,pp. $11-$ 110 .

${ }^{2}$ Butters, J. N., and Leendertz, J. A., "Speckle Pattern and Holographic Techniques in Engineering Metrology," Optics and Laser Technology, Vol. 3 , No. 1, 1971, pp. 26-30.

${ }^{3}$ Rastogi, P. K., Holographic Interferometry, Springer-Verlag, Berlin, 1994, pp. 7-32.

${ }^{4}$ Jones, R., and Wykes, C., Holographic and Speckle Interferometry, Cambridge Univ. Press, Cambridge, England, U.K., 1989, pp. 165-196.

${ }^{5} \mathrm{~L} \phi$ kberg, O. J., and Hogmoen, K., "Use of Modulated Reference Wave in Electronic Speckle Pattern Interferometry," Journal of Physics E: Scientific Instruments, Vol. 9, No. 10, 1976, pp. 847-851.

${ }^{6}$ Creath, K., and Slettemoen, G. A., "Vibration-Observation Techniques for Digital Speckle-Pattern Interferometry," Journal of the Optical Society of America A, Vol. 12, No. 10, 1985, pp. 1629-1636.

${ }^{7} \mathrm{~L} \phi$ kberg, O. J., "Mapping of In-Plane Vibration Modes by Electronic Speckle Pattern Interferometry," Optical Engineering, Vol. 24, No. 2, 1985 , pp. 356-359.

${ }^{8}$ Shellabear, M. C., and Tyrer, J. R., "Application of ESPI to ThreeDimensional Vibration Measurement," Optics and Lasers in Engineering, Vol. 15, No. 1, 1991, pp. 43-56.

${ }^{9}$ Doval, A. F., Fernández, J. L., Pérez-Amor, M. J., Valera, D. R., and Jones, J. D. C., "Contrast Enhanced and Phase Controlled Stroboscopic Additive Fiber Optic TV-Holography for Whole Field Out-of-Plane Vibration Analysis," Optics and Lasers in Engineering, Vol. 25, No. 4-5, 1996 , pp. 323-342.

${ }^{10}$ Wang, W. C., Hwang, C. H., and Lin, S. Y., "Vibration Measurement by the Time-Averaged Electronic Speckle Pattern Interferometry Methods," Applied Optics, Vol. 35, No. 22, 1996, pp. 4502-4509.

${ }^{11}$ Dimitriadis, E. K., Fuller, C. R., and Rogers, C. A., "Piezoelectric Actuators for Distributed Vibration Excitation of Thin Plates," Journal of Vibration and Acoustics, Vol. 113, No. 1, 1991, pp. 100-107.

${ }^{12}$ Berlincourt, D. A., Curran, D. R., and Jaffe, H., "Piezoelectric and Piezomagnetic Materials and Their Function in Transducers," Physical Acoustic, Vol. 1, No. A, 1964, pp. 169-270.

${ }^{13}$ Zelenka, J., Piezoelectric Resonators and Their Application, Academia, Prague, 1986, pp. 189-241.

${ }^{14}$ Tiersten, H. F., Linear Piezoelectric Plate Vibrations, Plenum, New York, 1969, pp. 33-61.

${ }^{15}$ Kharouf, N., and Heyliger, P. R., "Axisymmetric Free Vibration of Homogeneous and Laminated Piezoelectric Cylinders," Journal of Sound and Vibration, Vol. 174, No. 4, 1994, pp. 539-561.

${ }^{16}$ Heyliger, P., "Traction-Free Vibration of Layered Elastic and Piezoelectric Rectangular Parallelepipeds," Journal of the Acoustical Society of Amercia, Vol. 107, No. 3, 2000, pp. 1235-1245.

${ }^{17}$ Adelman, N. T., and Stavsky, Y., "Axisymmetric Vibrations of Radially Polarized Piezoelectric Ceramic Cylinders," Journal of Sound and Vibration, Vol. 38, No. 2, 1975, pp. 245-254.

${ }^{18}$ Adelman, N. T., and Stavsky, Y., "Vibrations of Radially Polarized Composite Piezoceramic Cylinders and Disks," Journal of Sound and Vibration Vol. 43, No. 1, 1975, pp. 37-44.

${ }^{19}$ Heyliger,P. R., and Ramirez, G., "Free Vibrations of Laminated Circular Piezoelectric Plates and Discs," Journal of Sound and Vibration, Vol. 229, No. 4, 2000, pp. 935-956.

${ }^{20}$ Migliori, A., and Sarrao, J. L., Resonant Ultrasound Spectroscopy: Applicationsto Physics, Materials Measurements, and Nondestructive Evaluation, Wiley, New York, 1997, pp. 119-136.

${ }^{21}$ Shaw, E. A. G., "On the Resonant Vibrations of Thick Barium Titanate Disks,"Journal of the Acoustical Society of Amercia, Vol. 28, No. 1, 1956, pp. 38-50

${ }^{22}$ Koyuncu, B., "The Investigation of High Frequency Vibration Modes of PZT-4 Transducers Using ESPI Techniques with Reference Beam Modulation," Optics and Lasers in Engineering, Vol. 1, No. 1, 1980, pp. 37-49.

${ }^{23} \mathrm{Ma}$, C. C., and Huang, C. H., "The Investigation of Three-Dimensional Vibration for Piezoelectric Rectangular Parallelepipeds Using the AF-ESPI Method," IEEE Transactions on Ultrasonics, Ferroelectrics, and Frequency Control, Vol. 48, No. 1, 2001, pp. 142-153.

${ }^{24}$ Huang, C. H., and Ma, C. C., "Vibration Characteristics for Piezoelectric Cylinders Using Amplitude-Fluctuation Electronic Speckle Pattern Interferometry," AIAA Journal, Vol. 36, No. 12, 1998, pp. 2262-2268.

${ }^{25}$ Cloud, G. L., Optical Methods of Engineering Analysis, Cambridge Univ. Press, New York, 1998, pp. 453-476.

${ }^{26}$ Rogacheva, N. N., The Theory of Piezoelectric Shells and Plates, CRC Press, Boca Raton, FL, 1994, pp. 3-14.

${ }^{27}$ Hibbit, H. D., Karlsson, B. J., and Sorensen, A., "ABAQUS User's Manual," Ver. 5.5, Hibbit, Karlsson, and Sorensen, Inc., Pawtucket, RI, 1995.

A. Berman Associate Editor 